\title{
GSTZ1 sensitizes hepatocellular carcinoma cells to sorafenib-induced ferroptosis via inhibition of NRF2/GPX4 axis
}

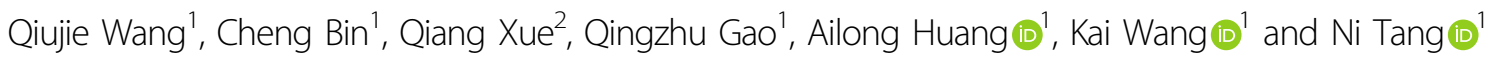

\begin{abstract}
Increasing evidence supports that ferroptosis plays an important role in tumor growth inhibition. Sorafenib, originally identified as an inhibitor of multiple oncogenic kinases, has been shown to induce ferroptosis in hepatocellular carcinoma (HCC). However, some hepatoma cell lines are less sensitive to sorafenib-induced ferroptotic cell death. Glutathione S-transferase zeta 1 (GSTZ1), an enzyme in the catabolism of phenylalanine, suppresses the expression of the master regulator of cellular redox homeostasis nuclear factor erythroid 2-related factor 2 (NRF2). This study aimed to investigate the role and underlying molecular mechanisms of GSTZ1 in sorafenib-induced ferroptosis in HCC. GSTZ1 was significantly downregulated in sorafenib-resistant hepatoma cells. Mechanistically, GSTZ1 depletion enhanced the activation of the NRF2 pathway and increased the glutathione peroxidase 4 (GPX4) level, thereby suppressing sorafenib-induced ferroptosis. The combination of sorafenib and RSL3, a GPX4 inhibitor, significantly inhibited GSTZ1deficient cell viability and promoted ferroptosis and increased ectopic iron and lipid peroxides. In vivo, the combination of sorafenib and RSL3 had a synergic therapeutic effect on HCC progression in Gstz $1^{-1-}$ mice. In conclusion, this finding demonstrates that GSTZ1 enhanced sorafenib-induced ferroptosis by inhibiting the NRF2/ GPX4 axis in HCC cells. Combination therapy of sorafenib and GPX4 inhibitor RSL3 may be a promising strategy in HCC treatment.
\end{abstract}

\section{Introduction}

Hepatocellular carcinoma (HCC) is the fourth leading cause of cancer-related death worldwide ${ }^{1}$. In the early stages of HCC, curative treatment can be achieved with tumor ablation, resection, or liver transplantation ${ }^{2}$. However, the majority of HCC patients are already in the middle-late stage when diagnosed; thus, the optimal period for curative treatment is missed. Sorafenib, a multi-target kinase inhibitor, has been confirmed to

\footnotetext{
Correspondence: Ailong Huang (ahuang@cqmu.edu.cn) or

Kai Wang (wangkai@cqmu.edu.cn) or Ni Tang (nitang@cqmu.edu.cn)

'Key Laboratory of Molecular Biology for Infectious Diseases (Ministry of Education), Institute for Viral Hepatitis, Department of Infectious Diseases, The Second Affiliated Hospital, Chongqing Medical University, Chongqing, China ${ }^{2}$ Department of Hepatobiliary Surgery, The Second Affiliated Hospital of Chongqing Medical University, Chongqing, China

These authors contributed equally: Qiujie Wang, Cheng Bin, Qiang Xue Edited by A. Linkermann
}

prolong the survival of advanced HCC patients to 6.5 months in phase III trial ${ }^{3}$. Thus, it has been approved by the Food and Drug Agency as a first-line treatment for advanced HCC. However, several patients with advanced HCC have limited survival benefits due to acquired resistance to sorafenib, leading to a high recurrence rate ${ }^{4}$. Therefore, the mechanism of sorafenib resistance needs to be explored, and new molecular targets should be identified.

Ferroptosis is a newly described programmed form of cell death characterized by iron-dependent accumulation of lipid peroxides to lethal amounts, different from the traditional cell death forms of apoptosis, necroptosis, and autophagy $^{5}$. Growing evidence indicates that ferroptosis can be induced by inhibiting cystine/glutamate transporter (SLC7A11/xCT) activity, downregulating glutathione peroxidase 4 (GPX4), and accumulating iron and lipid

\section{(c) The Author(s) 2021}

\footnotetext{
(c) Open Access This article is licensed under a Creative Commons Attribution 4.0 International License, which permits use, sharing, adaptation, distribution and reproduction cc) in any medium or format, as long as you give appropriate credit to the original author(s) and the source, provide a link to the Creative Commons license, and indicate if changes were made. The images or other third party material in this article are included in the article's Creative Commons license, unless indicated otherwise in a credit line to the material. If material is not included in the article's Creative Commons license and your intended use is not permitted by statutory regulation or exceeds the permitted use, you will need to obtain permission directly from the copyright holder. To view a copy of this license, visit http://creativecommons.org/licenses/by/4.0/.
} 
reactive oxygen species $(\mathrm{ROS})^{6-8}$. Recent reports have shown that sorafenib could induce ferroptosis; thus, targeting ferroptosis to improve sorafenib therapy might be a new promising strategy for HCC treatment ${ }^{9-11}$.

Glutathione S-transferases (GSTs) are a class of phase II detoxification enzymes that catalyze the conjugation of glutathione (GSH) to endogenous or exogenous electrophilic compounds ${ }^{12}$. GSTs, including GSTM and GSTP $^{13-15}$, are involved in the development of chemotherapy resistance ${ }^{16,17}$. Glutathione S-transferase zeta 1 (GSTZ1) is an important member of the GST superfamily. It participates in the catabolism of phenylalanine/ tyrosine and catalyzes the isomerization of maleylacetoacetate to fumarylacetoacetate ${ }^{18}$. We previously found that GSTZ1 was poorly expressed in HCC, and GSTZ1 deficiency could lead to metabolite succinylacetone accumulation and thereby activate the nuclear factor erythroid 2-related factor 2 (NRF2) signaling pathway ${ }^{19,20}$. Considering the importance of GSTZ1 in the development and progression of HCC, GSTZ1 may be an anticancer hallmark for sorafenib resistance in HCC. Therefore, it is crucial to investigate the role of GSTZ1 in chemotherapeutic resistance and elucidate underlying mechanisms. In the present study, we investigated the role of GSTZ1 in sorafenib-induced ferroptosis in HCC cell lines and in Gstz1-knockout mice and determined the involved molecular mechanisms. Our study not only identifies a novel mechanism of sorafenib resistance but also suggests a novel link between GSTZ1 and ferroptosis.

\section{Results}

\section{GSTZ1 is downregulated in sorafenib-resistant HCC}

To investigate the molecular mechanism of sorafenib resistance in HCC, we generated sorafenib-resistant (SR) HCC cell lines in vitro. Resistance was achieved by gradually increasing the concentration of sorafenib in the medium over repeated passages ${ }^{21}$. Finally, resistant HepG2 and SNU449 cell lines were established. We confirmed the acquired resistance of these resistant cells, named HepG2-SR and SUN449-SR, toward sorafenib by comparing them to the parental cells. The half-maximal inhibitory concentrations $\left(\mathrm{IC}_{50}\right)$ of HepG2-SR and SNU449-SR cells to sorafenib were 2-3 times higher than that of the parental cells at 17.09 and $15.43 \mu \mathrm{M}$, respectively (Fig. 1A). In addition, we evaluated the cell viability of sensitive and resistant cells treated with sorafenib over a series of time points or at different concentrations for $24 \mathrm{~h}$. We found that the SR cells became less sensitive to sorafenib (Fig. 1B, C), and the growth rate was remarkably increased (Fig. 1D). To verify the role of GSTZ1 in SR HCC, we comprehensively analyzed the expression levels of GSTZ1 in HepG2 cells and HepG2-SR cells in GEO dataset GSE62813. The results showed that GSTZ1 was significantly downregulated in SR cells (Fig. 1E).
Subsequently, we further validated the low levels of GSTZ1 expression in SR cell lines via quantitative reversetranscription polymerase chain reaction (qRT-PCR) and western blotting (Fig. 1F, G). Together, these data indicate that GSTZ1 may play a negative role in mediating sorafenib resistance in HCC cells.

\section{GSTZ1 knockout promotes sorafenib resistance in HCC}

To further evaluate whether GSTZ1 is related to sorafenib resistance in HCC, we found that overexpression of GSTZ1 through an adenovirus system ${ }^{19,20}$ (Fig. 2A) increased the sensitivity of HCC cells to sorafenib and inhibited cell proliferation via morphological observation (Fig. 2B). Conversely, knockout of GSTZ1 in HepG2 and SNU449 cells via the CRISPR/Cas9 system ${ }^{19,20}$ (Fig. 2C) decreased sorafenib sensitivity and weakened its growth inhibition effect (Fig. 2D). Next, we analyzed the cell viability of GSTZ1 overexpression (OE) and knockout (KO) cells treated with sorafenib over a series of time points or at different concentrations for $24 \mathrm{~h}$ by cell growth curve. As expected, GSTZ1 overexpression significantly enhanced the sensitivity of $\mathrm{HCC}$ cell lines to sorafenib (Fig. 2E, F). As expected, the $\mathrm{IC}_{50}$ value of GSTZ1-OE cells was decreased compared to that of the control groups, whereas the $\mathrm{IC}_{50}$ value of GSTZ1-KO groups was increased (Fig. 2G, H). Taken all together, our results showed that GSTZ1 deficiency enhanced sorafenib resistance in $\mathrm{HCC}$.

\section{GSTZ1 overexpression enhances sorafenib-induced ferroptosis in HCC}

Recent studies indicate that ferroptosis plays a key role in the chemoresistance of human cancers ${ }^{22-24}$. We confirmed that sorafenib-induced cell death in HCC cell lines was blocked by ferrostatin-1 (Fer-1, an inhibitor of ferroptosis), deferoxamine (DFO, an iron chelator), and $\mathrm{N}$ acetyl-L-cysteine (NAC, an antioxidant), but not by bafilomycin A1 (Baf-A1, an inhibitor of autophagy), ZVADFMK, and necrosulfonamide (NSA, an inhibitor of necroptosis). This suggested that ferroptosis, rather than apoptosis, is essential for sorafenib-induced cell death in HCC (Supplementary Fig. 1A, B), consistent with findings of the previous studies ${ }^{11,25,26}$. To determine whether GSTZ1 played a role in ferroptosis to reduce sorafenib resistance in HCC, transmission electron microscopy (TEM) was used to observe morphological changes in sorafenib-induced HCC cells with or without GSTZ1 depletion. GSTZ1-OE cells treated with sorafenib displayed smaller mitochondria, diminished or vanished mitochondria crista, and condensed mitochondrial membrane densities compared to parental cells, whereas GSTZ1-KO alleviated the abnormalities of mitochondrial morphology and cell death induced by sorafenib (Fig. 3A). To further verify this observation, we measured ROS, 


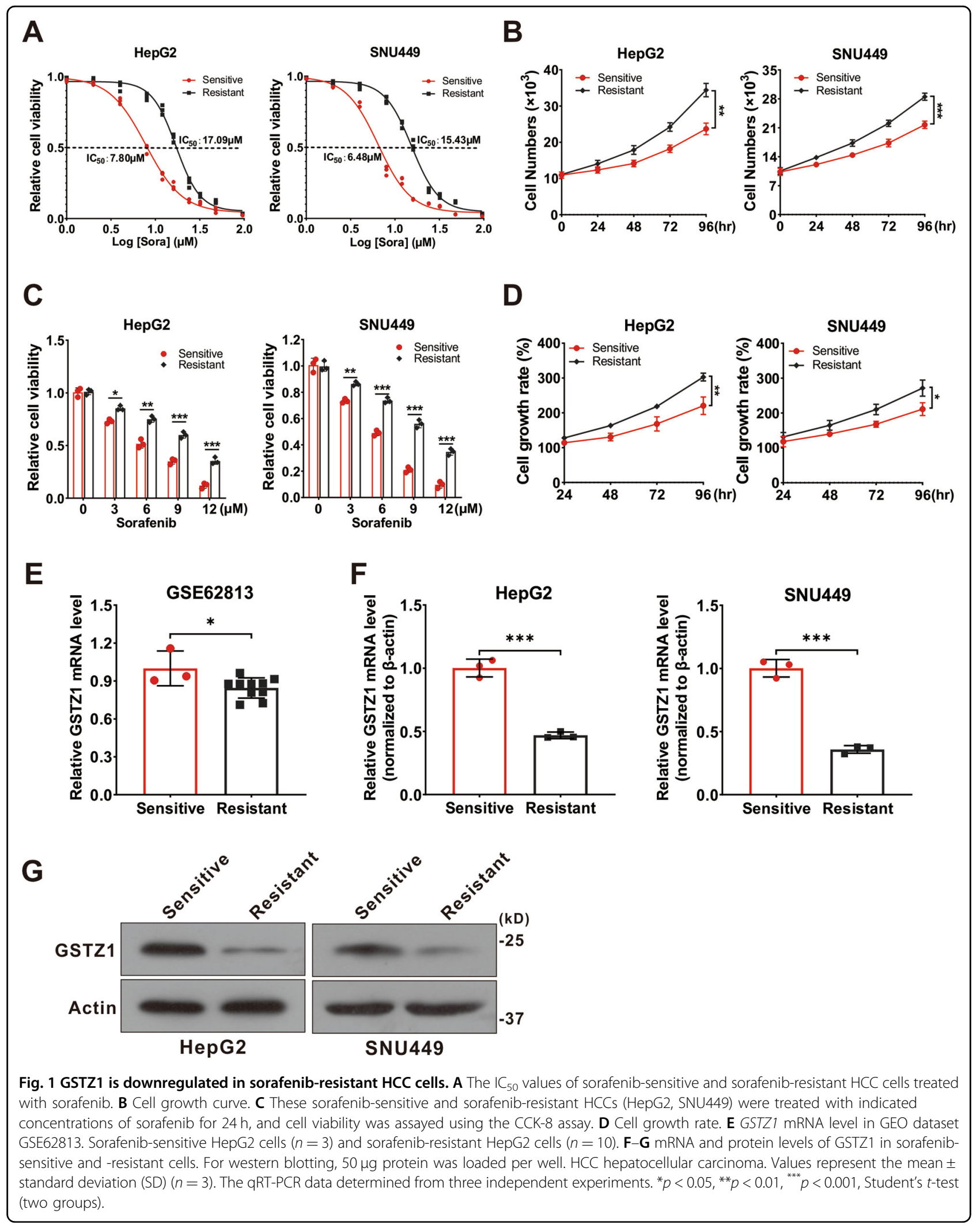



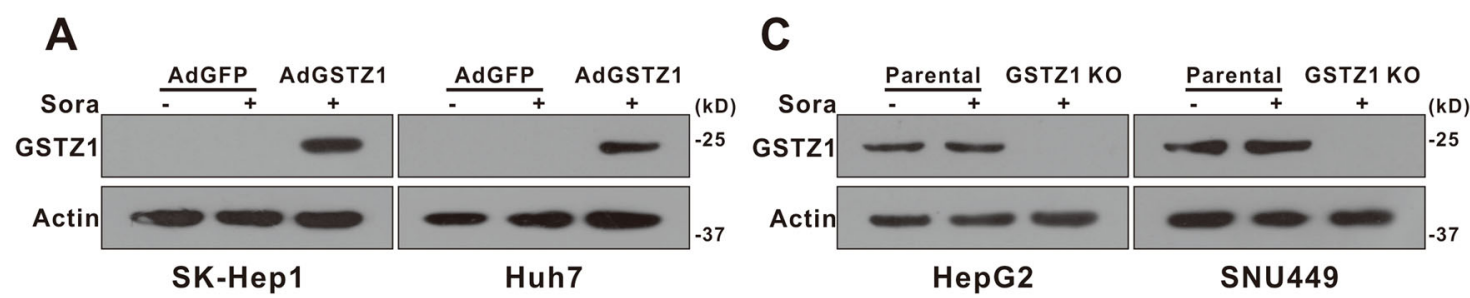

B

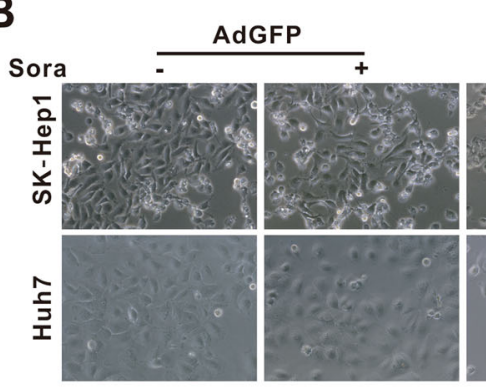

AdGSTZ1

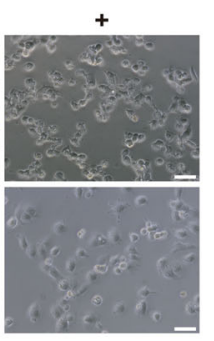

D
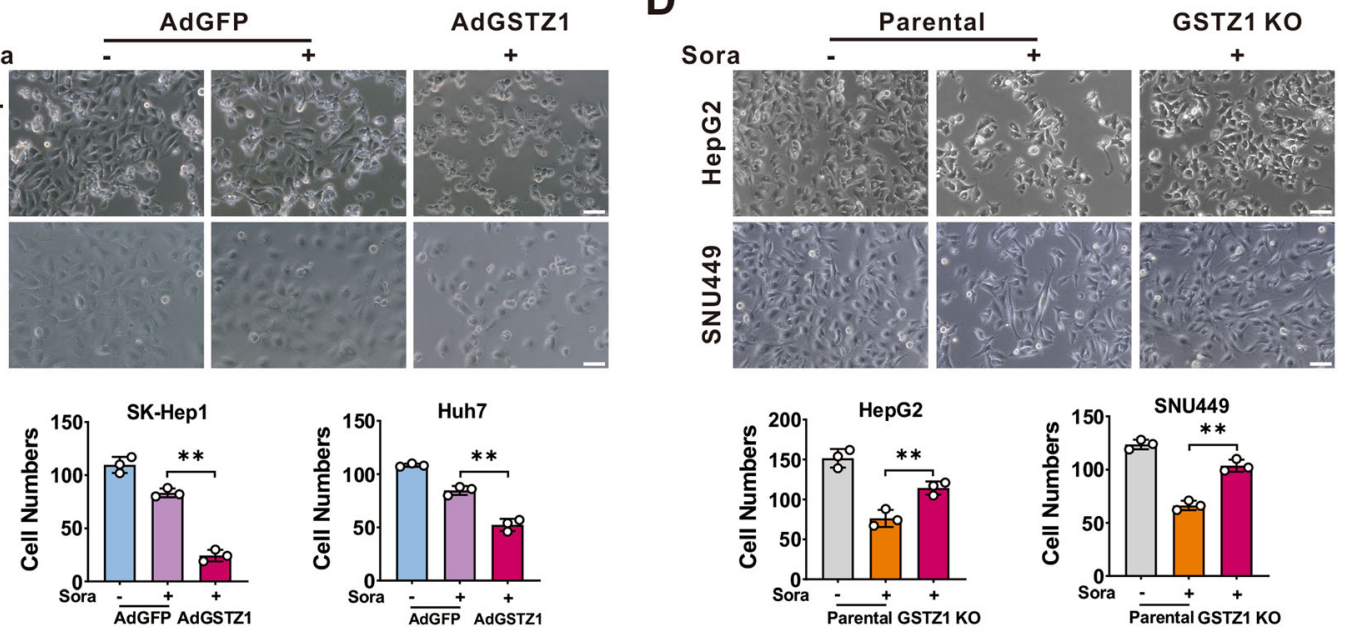

E

$\mathbf{F}$
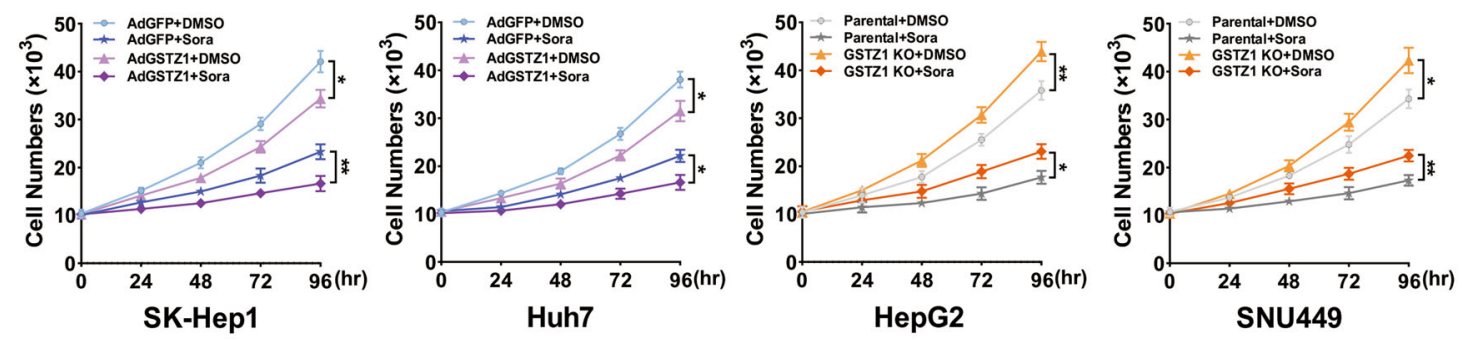

G

H

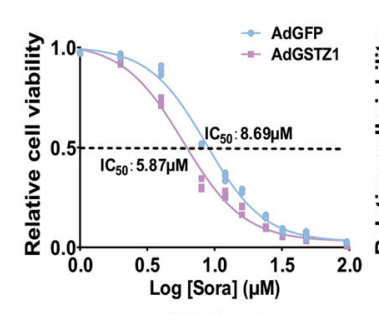

SK-Hep1

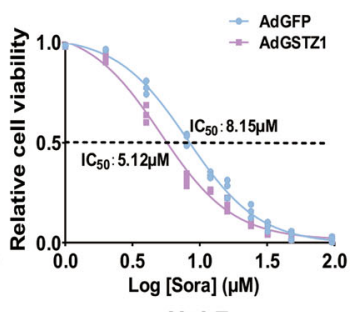

Huh7
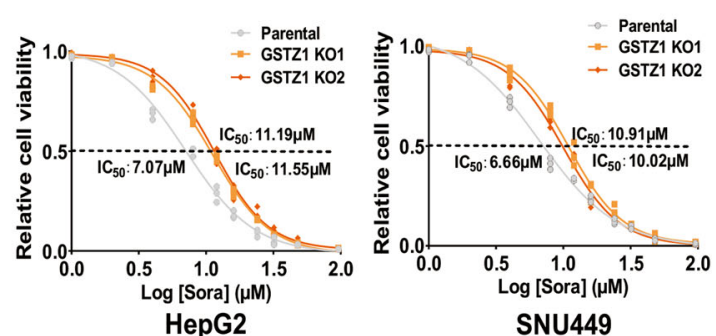

Fig. 2 GSTZ1 knockout promotes sorafenib resistance in HCC cells. A Overexpression of GSTZ1 was confirmed by immunoblot assay. B Morphological phase-contrast images (top) and quantification (bottom) of GSTZ1-OE cells after treatment with or without sorafenib (10 $\mu$ M) for $24 \mathrm{~h}$. C Knockout of GSTZ1 was confirmed by immunoblot assay. D Morphological phase-contrast images (top) and quantification (bottom) of GSTZ1$\mathrm{KO}$ cells after treatment with or without sorafenib $(10 \mu \mathrm{M})$ for $24 \mathrm{~h}$. Scale bar $=10 \mu \mathrm{m}$. E, F Cell growth curve. GSTZ1-OE (E) and GSTZ1-KO (F) cells were treated with or without sorafenib $(10 \mu \mathrm{M})$. G, H The IC 50 of GSTZ1-OE $(\mathbf{G})$ and GSTZ1-KO $(\mathbf{H})$ cells were determined using the CCK-8 assay. For western blotting, $50 \mathrm{\mu g}$ protein was loaded per well. DMSO: dimethyl sulfoxide, Sora: sorafenib. Values represent the mean $\pm \operatorname{SD}(n=3)$. ${ }^{*} p<0.05$, ** $p$ $<0.01$, Student's t-test (two groups). 


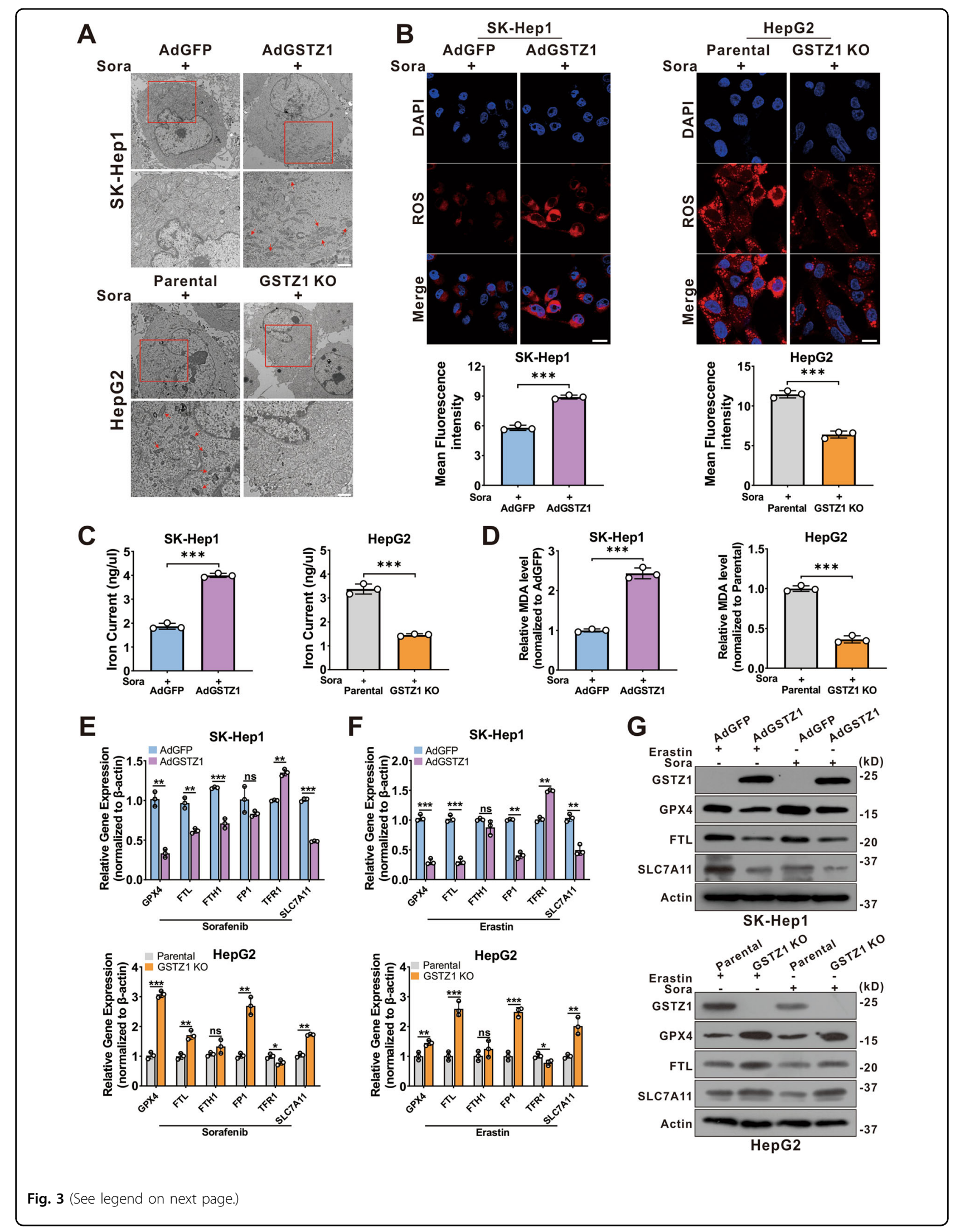


(see figure on previous page)

Fig. 3 GSTZ1 overexpression enhances sorafenib-induced ferroptosis in HCC. A Representative TEM images of the mitochondrial morphology in GSTZ1-OE SK-Hep1 and GSTZ1-KO HepG2 cells treated with $10 \mu \mathrm{M}$ sorafenib for $24 \mathrm{~h}$. Red arrows indicate mitochondria. Scale bar $=1 \mu \mathrm{m}$. B Representative images (top) and quantification (bottom) of ROS level in GSTZ1-OE and GSTZ1-KO cells treated with sorafenib for $24 \mathrm{~h}$. Scale bar $=$ $20 \mu \mathrm{m}$. C, D The intracellular iron (C) and MDA (D) levels in GSTZ1-OE and GSTZ1-KO cells treated with sorafenib for $24 \mathrm{~h}$. E-G mRNA and protein levels of target genes associated with ferroptosis in GSTZ1-OE and GSTZ1-KO cells treated with sorafenib or erastin, determined via qRT-PCR (E, F) and western blotting $(\mathbf{G})$, respectively. For western blotting, $50 \mu \mathrm{g}$ protein was loaded per well. Values represent the mean $\pm S D(n=3)$. The qRT-PCR data determined from three independent experiments. ns: no significant difference, ${ }^{*} p<0.05,{ }^{* *} p<0.01,{ }^{* *} p<0.001$, Student's t-test (two groups).

iron, and lipid peroxidation levels, which are the primary cause of ferroptosis ${ }^{27}$, after interference with GSTZ1 expression. Results showed that GSTZ1 overexpression significantly increased ROS, iron, and MDA levels in sorafenib-induced HCC cell lines (Fig. 3B-D and Supplementary Fig. 1C, D), whereas GSTZ1 knockout decreased their levels. In addition, we assessed mRNA and protein expression levels of ferroptosis-associated genes. Results showed that GSTZ1-OE decreased the expression levels of ferroptosis-related genes in sorafenib or erastin (an inducer of ferroptosis)-treated hepatoma cells, including GPX4, FTL, and SLC7A11. In contrast, GSTZ1$\mathrm{KO}$ increased the levels of these above genes (Fig. 3E-G and Supplementary Fig. 1E, F). Interestingly, ferroptosisassociated genes were also enhanced in HepG2-SR and SNU449-SR cells (Fig. 4A, B), which had relatively lower levels of GSTZ1 than the parental cells. Meanwhile, GSTZ1 overexpression in drug-resistant cells reduced the levels of ferroptosis-related genes (Fig. 4C), increased the accumulation of iron and MDA levels (Fig. 4D, E), decreased the intracellular GSH level (Fig. 4F), and enhanced the inhibition of sorafenib to resistant cells (Fig. 4G). The GSTZ1 Ser14 $\rightarrow$ Ala mutation has the greatest detrimental effect on GSTZ1 activity ${ }^{28}$. We then generated GSTZ-KO and SR cells expressing S14A GSTZ1 (Supplementary Fig. 2A, B) and found that upon sorafenib stimulation, iron and MDA levels were reduced in the cells expressing S14A GSTZ1 compared to wildtype (WT)-transduced cells (Supplementary Fig. 2C-F). This suggests that GSTZ1 S14A failed to sensitize hepatoma cells to sorafenib-induced ferroptosis (Supplementary Fig. 2G, H). To further identify the role of ferroptosis in sorafenib resistance caused by GSTZ1 deficiency, we examined the curative effects of sorafenib by interfering with the ferroptosis pathway. We observed that ferrostatin-1 inhibited sorafenib-induced GSTZ1-OE cell death, whereas erastin promoted GSTZ1-KO cell death (Fig. 4H). Together, these results suggested that GSTZ1 increased the sensitivity of hepatoma cells to sorafenib by inducing ferroptosis.

\section{GSTZ1 sensitizes hepatoma cells to sorafenib-induced ferroptosis through the NRF2 signaling pathway}

Previous studies have shown that GSTZ1 deficiency leads to the accumulation of endogenous metabolite succinylacetone, contributing to NRF2 activation via alkylating Kelch-like ECH-associated protein 1 (KEAP1) cysteine residues ${ }^{19,20}$. Activation of NRF2 pathway plays a critical role in protecting HCC cells against sorafenib-induced ferroptosis ${ }^{26}$. To verify whether GSTZ1 regulated sorafenib-induced ferroptosis through the NRF2 signaling pathway, we blocked the NRF2 pathway using brusatol (an inhibitor of NRF2) or Flag-tagged KEAP1 ${ }^{29}$ (a cytosolic inhibitor of NRF2) in GSTZ1-KO cells. We then observed the characteristic indicators related to ferroptosis, including iron, MDA, ROS, and 4-HNE levels. The results demonstrated that NRF2 inhibition significantly increased the accumulation of these indicators in GSTZ1-KO cells (Fig. 5A-D right, Fig. 5E, F bottom, and Supplementary Fig. 3A-C bottom), whereas NRF2 activation using tertiary butylhydroquinone (tBHQ, an activator of NRF2) and Myc-tagged NRF2 yielded opposite results in GSTZ1OE cells (Fig. 5A-D left, Fig. 5E-F top and Supplementary Fig. 3A-C top). Moreover, the morphological images also indicated that NRF2 inhibition increased the efficiency of sorafenib for growth inhibition in GSTZ1-depleted HCCs, whereas NRF2 activation decreased that in GSTZ1-OE cells (Fig. 6A). Importantly, western blotting revealed that the expression of ferroptosis-related proteins changed accordingly in GSTZ1-OE and -KO cells when treated with tBHQ or brusatol (Fig. 6B and Supplementary Fig. 3D). The above data suggested that GSTZ1 depletion alleviated sorafenib-induced ferroptosis via activation of the NRF2 pathway.

As GPX4 is involved in ferroptosis and a transcriptional target gene of $\mathrm{NRF}^{30,31}$, we utilized RSL3 to further examine whether ferroptosis is involved in the sensitivity of HCC cells to sorafenib. Interestingly, simultaneous treatment with sorafenib and RSL3 increased the accumulation of iron and MDA levels, decreased the intracellular GSH level, enhanced sorafenib-induced ferroptosis, and dramatically inhibited cell growth compared to sorafenib or RSL3 alone in GSTZ1-KO and SR cells (Fig. 6C-F). Collectively, these findings indicated that the inhibition of NRF2 could markedly sensitize GSTZ1-deficient hepatoma cells to sorafenib treatment. Furthermore, targeting GPX4 also improved the response of hepatoma cells to sorafenib. 

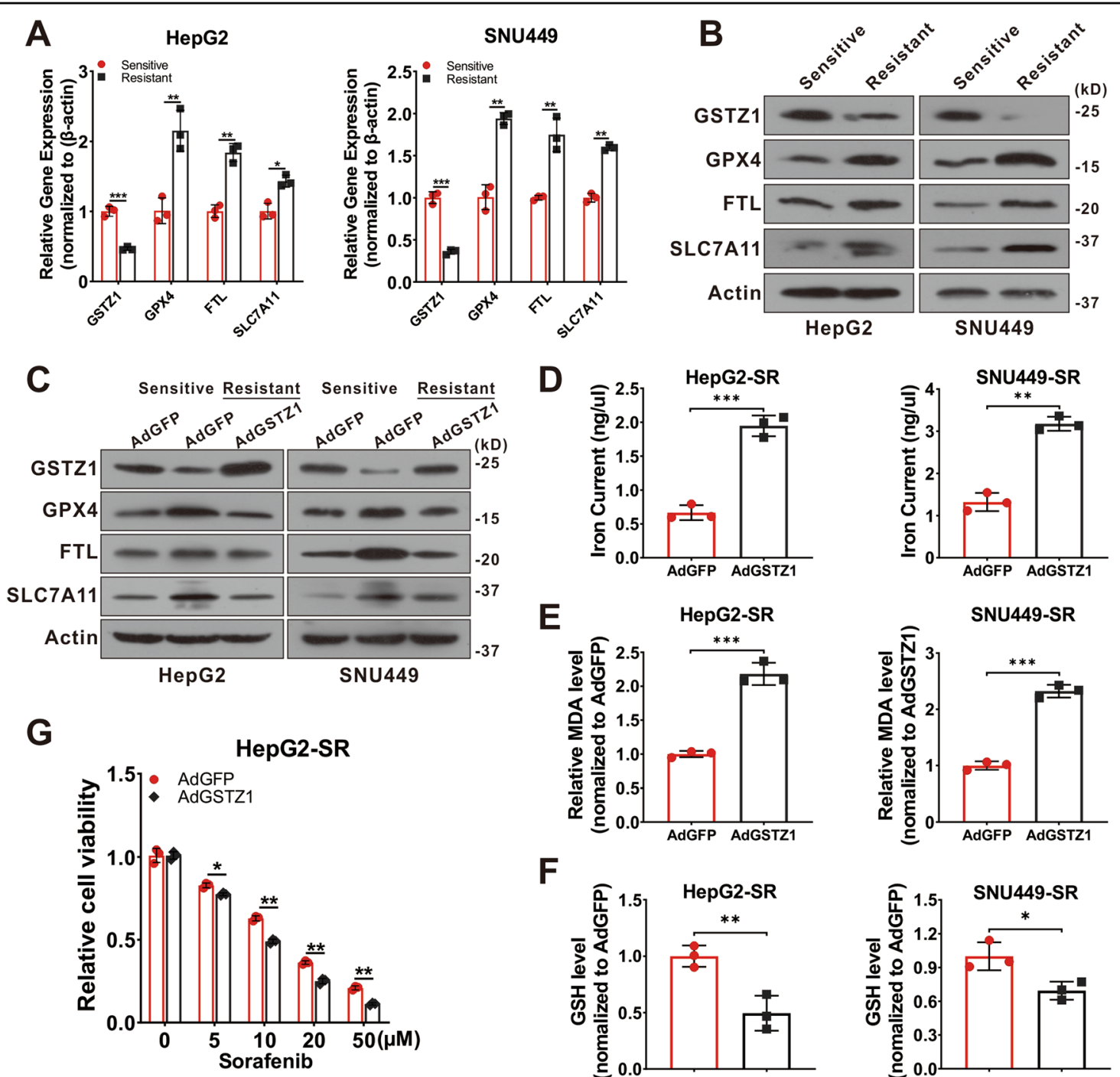

E

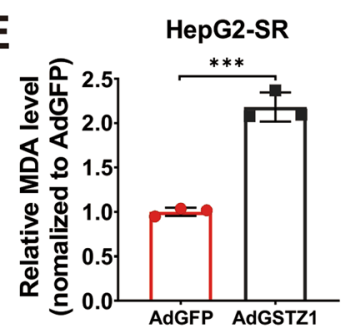

SNU449-SR

$\mathbf{F}$
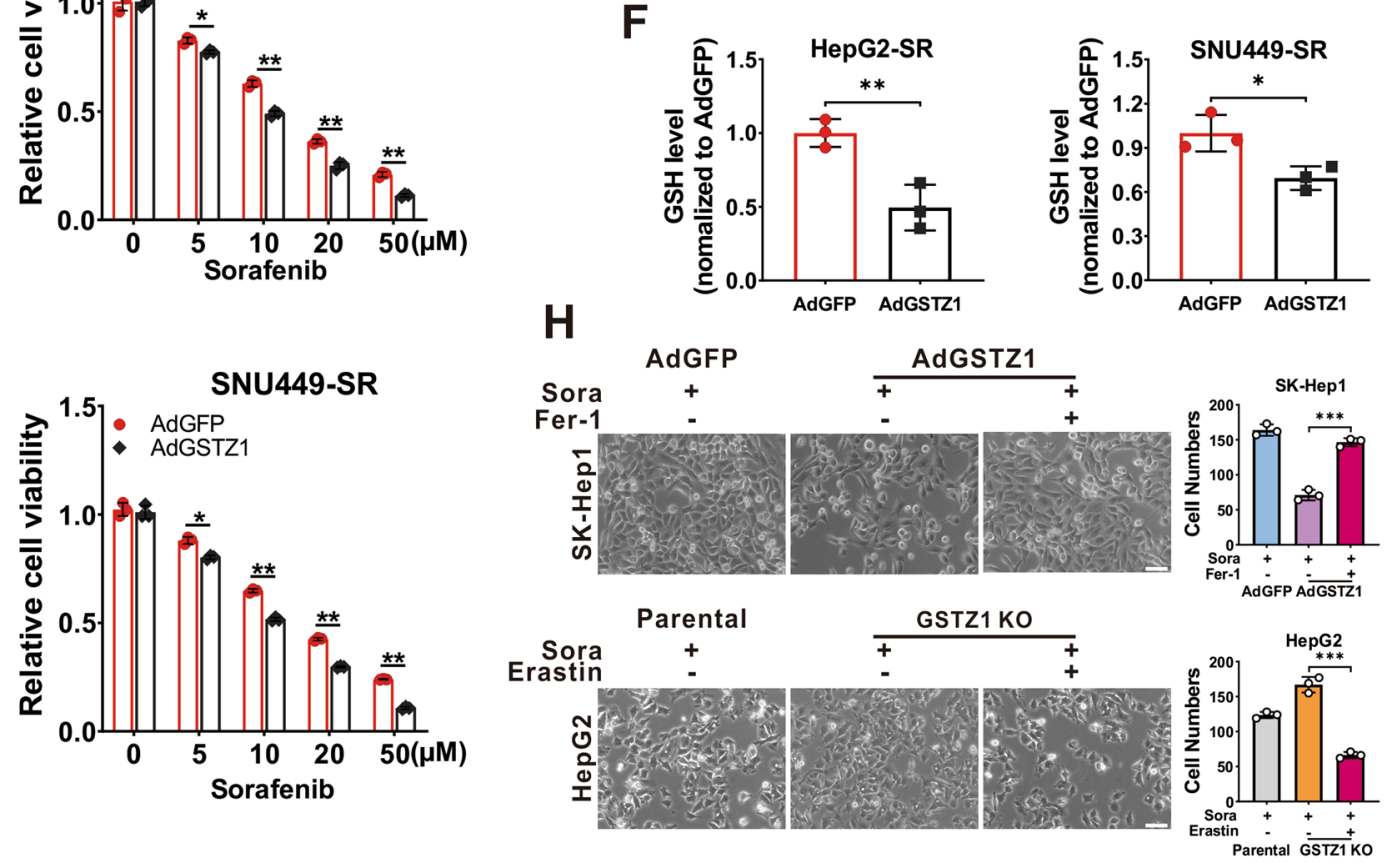

Fig. 4 GSTZ1 overexpression sensitizes hepatoma cells to sorafenib-induced ferroptosis. A, B mRNA (A) and protein (B) levels of ferroptosisrelated genes in sorafenib-sensitive and -resistant cells were assayed using qRT-PCR and western blotting, respectively. C Western blotting for assessment of protein levels of ferroptosis-related genes in sorafenib-resistant HCCs with adenoviruses expressing GFP (AdGFP) or GSTZ1 (AdGSTZ1). D-F Iron (D), MDA (E) and GSH (F) levels in GSTZ1-OE sorafenib-resistant cells. G Cell viability was determined using CCK-8 assay. H Morphology (left) and quantification (right) of indicated HCC cells treated with sorafenib $(10 \mu \mathrm{M}$ for $24 \mathrm{~h})$ alone or in combination with Fer-1 (1 $\mu \mathrm{M}$ for $24 \mathrm{~h})$ or erastin $(10 \mu \mathrm{M}$ for $24 \mathrm{~h})$. Scale bar $=10 \mu \mathrm{m}$. For western blotting, $50 \mathrm{\mu g}$ protein was loaded per well. Fer-1: ferrostatin-1, SR sorafenib resistant. Values represent the mean \pm SD $(n=3)$. The qRT-PCR data determined from three independent experiments. ${ }^{*} p<0.05,{ }^{* *} p<0.01$, ${ }^{* *} p<0.001$, Student's $t$-test (two groups). 


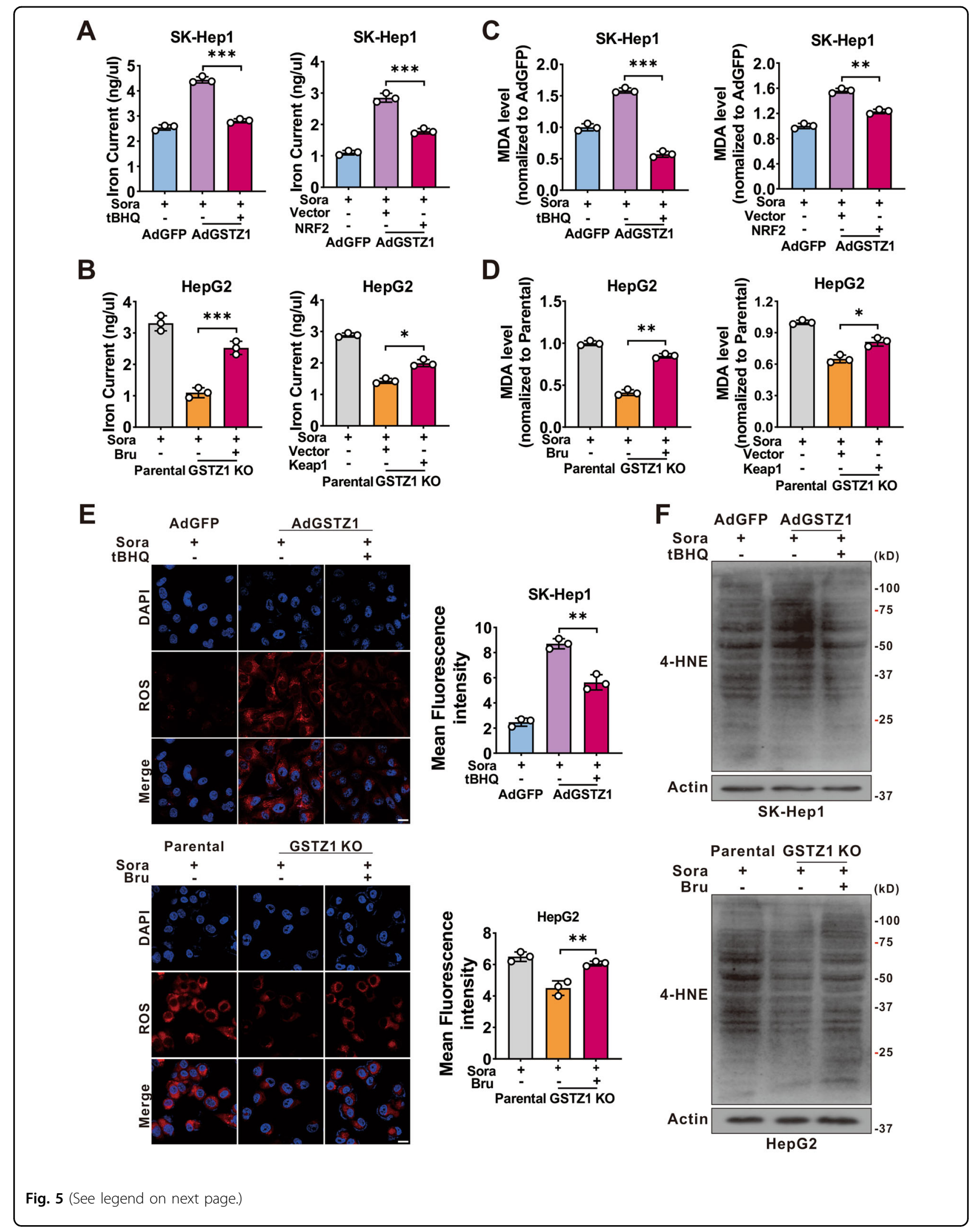


(see figure on previous page)

Fig. 5 GSTZ1 knockout cells are insensitive to sorafenib-induced ferroptosis through the activation of NRF2. A-D Levels of iron (A, B), and MDA (C, D) were assayed. GSTZ1-OE cells were treated with sorafenib alone or in combination with tBHQ (100 $\mu \mathrm{M}$ for $3 \mathrm{~h}$ ) (left). GSTZ1-KO cells were treated with sorafenib alone or in combination with Bru (40 nM for $24 \mathrm{~h}$ ) (left). Expressing Flag-KEAP1 plasmid was transfected into GSTZ1-OE cells with sorafenib treatment (right). Expressing Myc-NRF2 plasmid was transfected into GSTZ1-KO cells with sorafenib treatment (right). E Representative images (top) and quantification (bottom) of ROS level in GSTZ1-OE cells treated with sorafenib alone or in combination with tBHQ (top) and GSTZ1$\mathrm{KO}$ cells treated with sorafenib alone or in combination with Bru (bottom). Scale bar $=20 \mu \mathrm{m}$. F 4-HNE-induced protein modification was examined. For western blotting, $50 \mathrm{\mu g}$ protein was loaded per well. tBHQ: tertiary butylhydroquinone, Bru brusatol, 4-HNE 4-hydroxy-2-nonenal. Values represent the mean $\pm \mathrm{SD}(n=3) .{ }^{*} p<0.05,{ }^{* *} p<0.01,{ }^{* * *} p<0.001$, Student's $t$-test (two groups).

\section{RSL3 enhances the anticancer activity of sorafenib in} Gstz1 ${ }^{-1-}$ mice

To further investigate the role of GSTZ1 in mediating sorafenib resistance in HCC progression in vivo, we established the mouse model of liver cancer induced by $\mathrm{DEN} / \mathrm{CCl}_{4}$ as described previously ${ }^{20}$ and drug administration with three regimens: DMSO, sorafenib $(30 \mathrm{mg} / \mathrm{kg}$, every 2 days for 4 weeks), RSL3 (10 mg/kg, every 2 days for 4 weeks) (Fig. 7A). Compared with WT mice, Gstz1 knockout significantly reduced the inhibitory effects of sorafenib treatment in vivo than that in WT mice, as indicated by the increased tumor sizes, number of tumor nodules, and a higher level of alanine aminotransferase (ALT) in serum (Fig. 7B-E). Moreover, sorafenib combined with RSL3 had a more significant protective effect on tumorigenesis in Gstz1-/- mice than sorafenib alone. To substantiate the role of GSTZ1 in regulating ferroptosis-mediated sorafenib resistance in vivo, we detected the levels of iron, 4-HNE modification, MDA, ROS, and ferroptosis-associated gene expression in the liver tumor tissues. Consistent with the results in vitro, Gstz1 knockout decreased the sensitivity of HCC to sorafenib by weakening ferroptosis (Fig. 7B-J and Supplementary Fig. 4A). Meanwhile, RSL3/sorafenib combination treatment reduced drug resistance caused by GSTZ1 depletion (Fig. 7B-J). Furthermore, the histological analysis indicated that combination treatment groups exhibited weaker immunoreactivity for GPX4 and Ki67. RSL3 significantly enhanced the inhibitory effects of sorafenib on cell proliferation in Gstz1 $1^{-/-}$mice, which was highly consistent with our in vitro results (Fig. 7K). These results indicate that targeting the NRF2/GPX4 axis using RSL3 significantly enhances sorafenib-induced ferroptosis and inhibits hepatocarcinogenesis in vivo.

\section{Discussion}

The incidence of HCC continues to increase globally, and $\mathrm{HCC}$ remains to have high incidence and mortality rates $^{32}$. Sorafenib resistance remains a treatment challenge in HCC and leads to poor prognosis ${ }^{33}$. Therefore, the comprehensive elucidation of the underlying mechanism of sorafenib resistance in HCC may improve the curative effect of chemotherapy and guide the clinical medication. Herein, we found that GSTZ1 depletion activated the NRF2/GPX4 pathway and inhibited sorafenib-induced cell death, accompanied by the compromised accumulation of iron level, lipid peroxidation, and subsequent ferroptosis. Hence, blocking the NRF2/ GPX4 pathway to enhance the anticancer activity of sorafenib by inducing ferroptosis represents a promising therapeutic strategy for the treatment of HCC (Fig. 8).

GSTs are phase II detoxification enzymes that play important roles in protecting cellular macromolecules from both oxidative stress and carcinogenic electrophiles $^{12}$. The major roles of GSTs in the detoxification of xenobiotics predict their important role in drug resistance. Tumor cells may develop resistance to alkylating anticancer drugs by increasing the levels of GSTs ${ }^{16}$. Several subclasses in the GST family contribute to chemoresistance in various cancers ${ }^{15,34-39}$. GSTM1 was reported to be a predictive biomarker for the efficacy of chemotherapy in breast cancer and ovarian cancer ${ }^{15,34}$. GSTP expression was also correlated with platinum drug chemosensitivity and prognosis in ovarian cancer, pancreatic ductal adenocarcinoma, and lung cancer ${ }^{35-38}$. As a member of the GST family, GSTZ1 plays a similar detoxification role, but it is independently characterized as a maleylacetoacetate isomerase (MAAI), which is essential for phenylalanine metabolism ${ }^{18}$. We previously reported that GSTZ1 is downregulated in HCC, leading to increased accumulation of the carcinogenic metabolite succinylacetone and activation of the NRF2/IGF1R pathways through inactivation of KEAP ${ }^{19}$. The current study demonstrates that GSTZ1 is also downregulated in SR HCC cells. Furthermore, re-expression of GSTZ1 enhances the sensitivity of HCC cells to sorafenib treatment, indicating that GSTZ1 plays a negative regulatory role in sorafenib resistance.

Iron is an essential element for the synthesis of iron sulfur clusters, serving an important role in numerous cellular processes ${ }^{40}$. Cancer cells exhibit a higher dependence on iron than normal cells ${ }^{41}$, making them more susceptible to iron-catalyzed necrosis. This form of cell death was first defined as ferroptosis in $2012^{5}$ and was characterized by the accumulation of lipid peroxidation products and lethal ROS derived from iron metabolism ${ }^{6,27}$. An increasing number of small molecule compounds (e.g., erastin) or clinical drugs (e.g., sulfasalazine) 

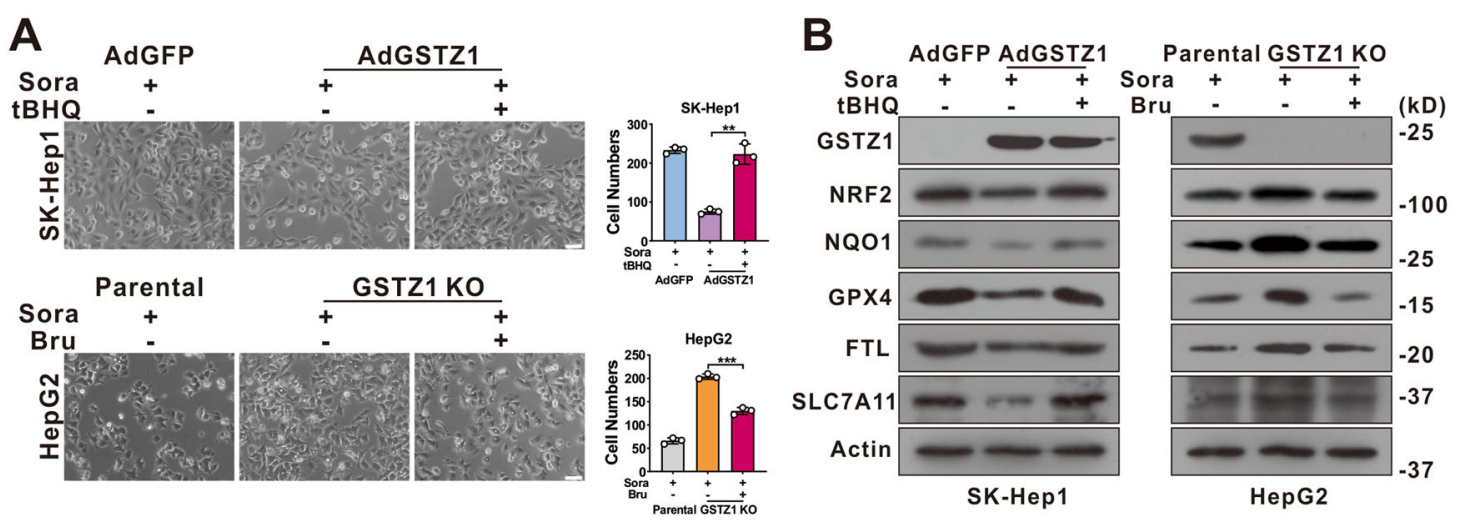

C
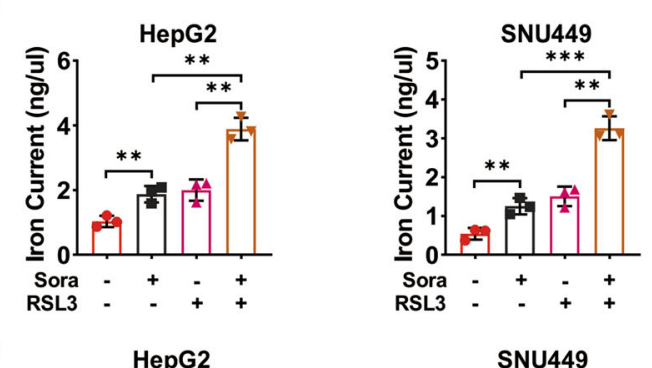

HepG2-SR
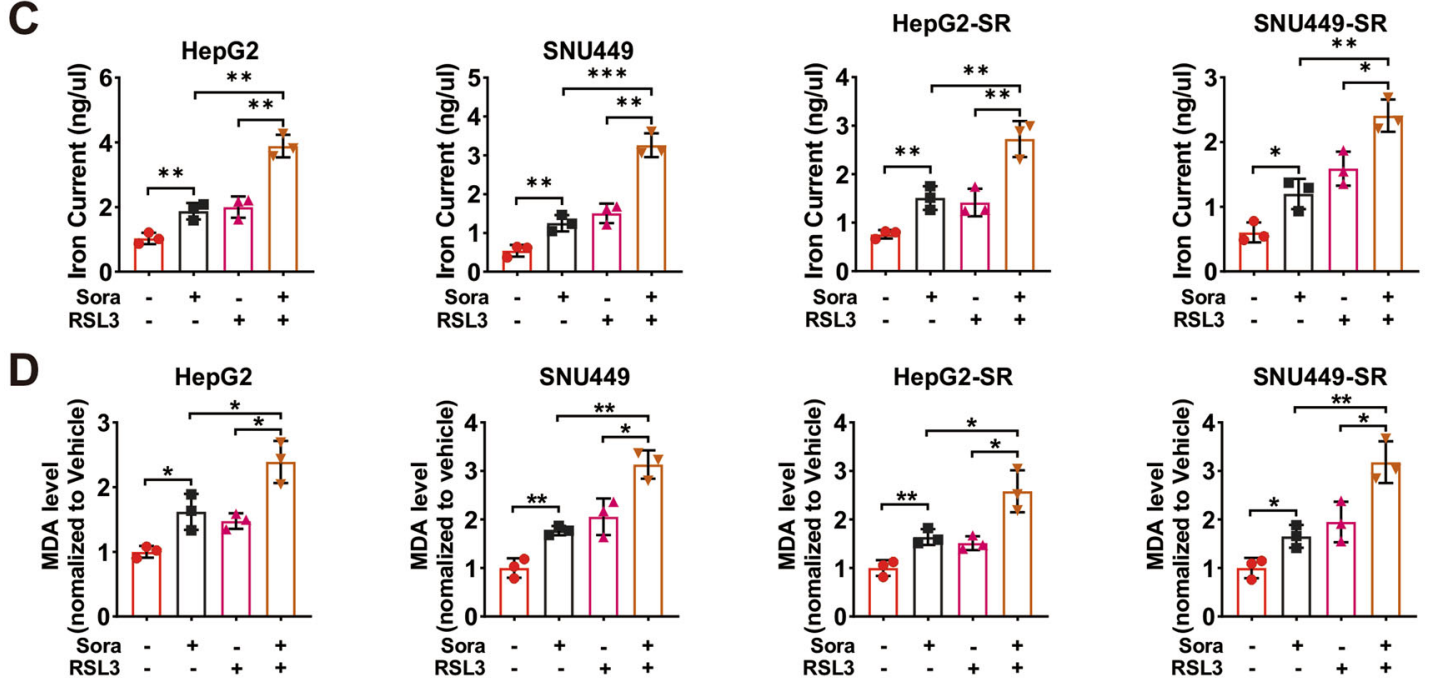

E

\section{HepG2}
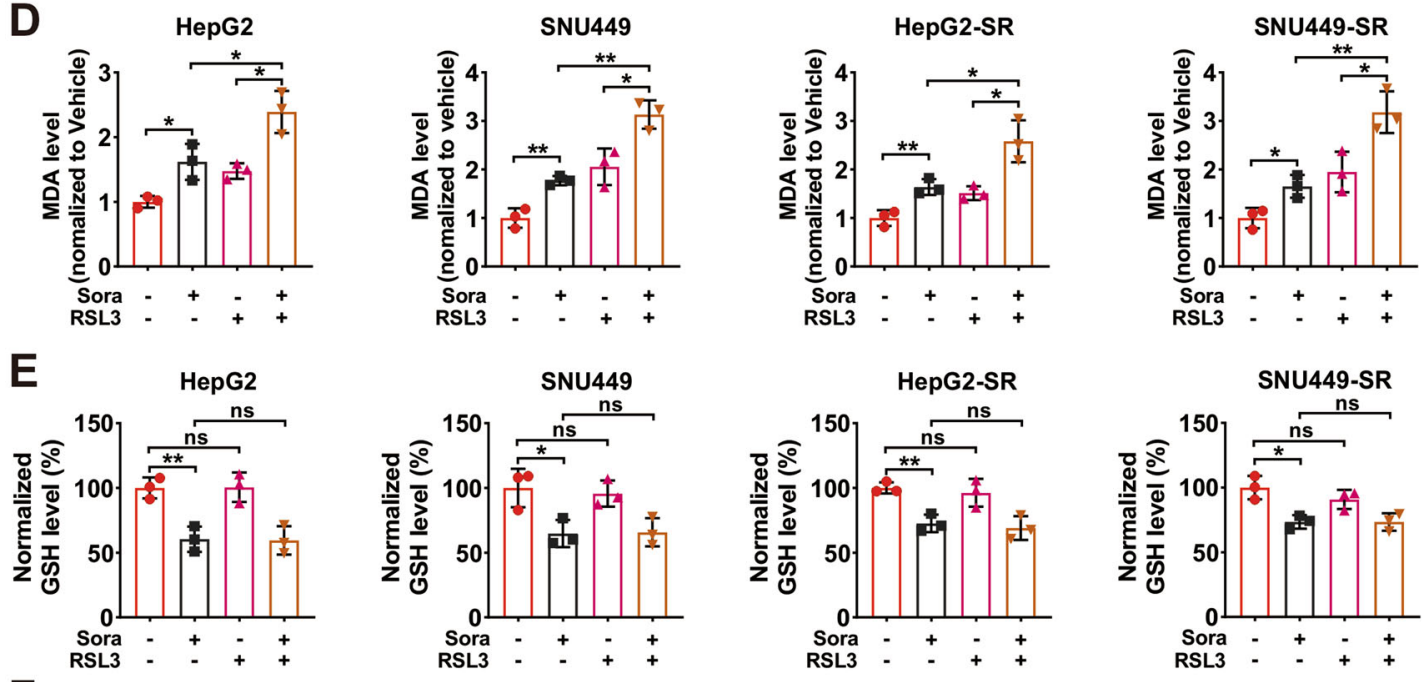

$\mathbf{F}$
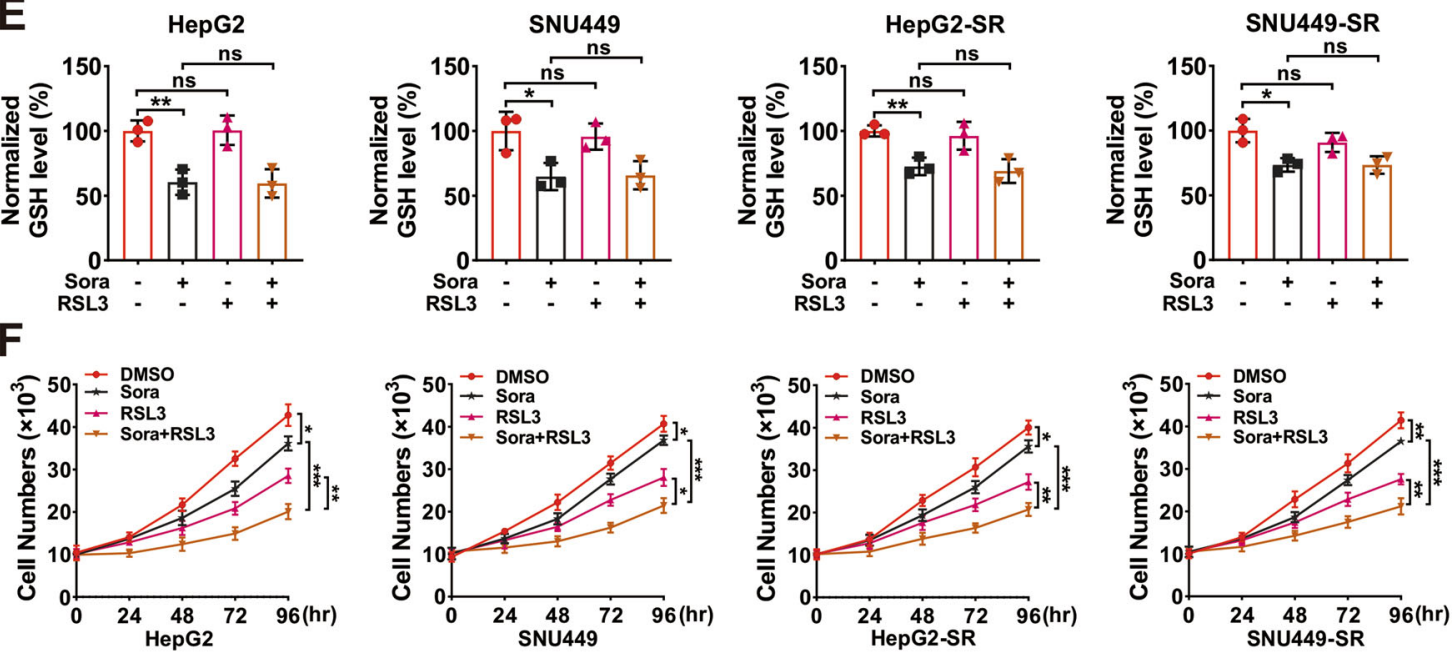

Fig. 6 RSL3 enhances the sensitivity of GSTZ1-KO and sorafenib-resistant cells to sorafenib. A, B Morphological changes (A) and protein level (B) of ferroptosis-related genes. GSTZ1-OE SK-Hep1 cells were treated with sorafenib alone or in combination with tBHQ (top). GSTZ1-KO HepG2 cells were treated with sorafenib alone or in combination with Bru (bottom). Scale bar $=10 \mu \mathrm{m}$. C-E Iron (C), MDA (D), and GSH (E) levels in GSTZ1-KO and sorafenib-resistant cells treated with sorafenib alone or in combination with RSL3 (500 nM for $24 \mathrm{~h}$ ). F Cell growth curve of GSTZ1-KO and sorafenibresistant cells treated with sorafenib alone or in combination with RSL3. RSL3: Ras-selective lethal small molecule 3, tBHQ tertiary butylhydroquinone, Bru brusatol, DMSO dimethyl sulfoxide, Sora sorafenib, MDA malondialdehyde. Values represent the mean $\pm \operatorname{SD}(n=3) .{ }^{*} p<0.05,{ }^{* *} p<0.01,{ }^{* * *} p<$ 0.001 , Student's $t$-test (two groups). 
A

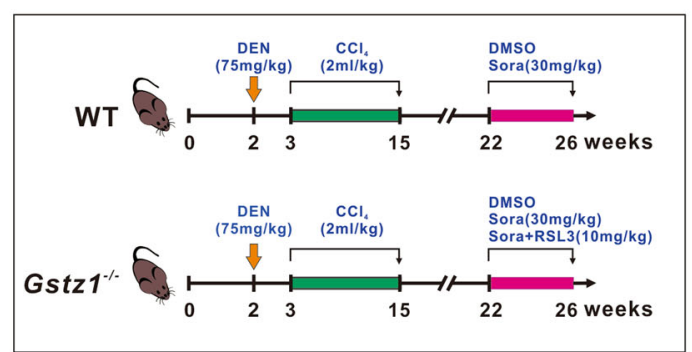

$\mathrm{C}_{\mathrm{g}}$

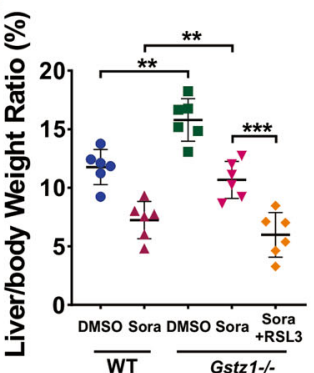

D

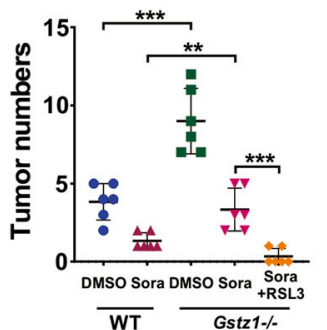

G

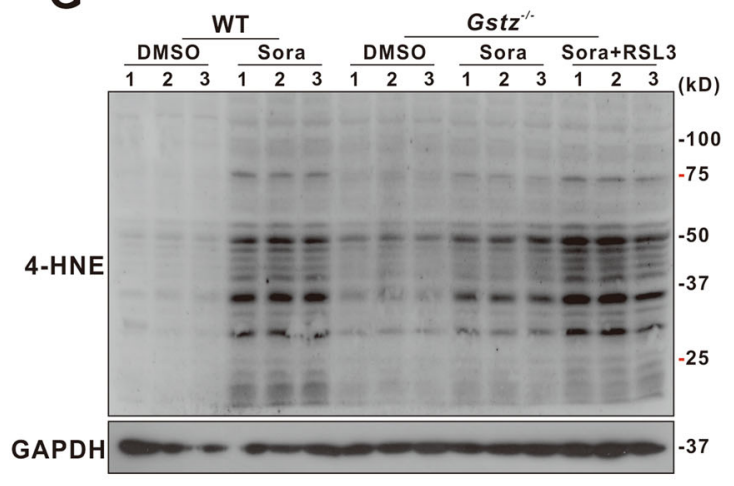

J
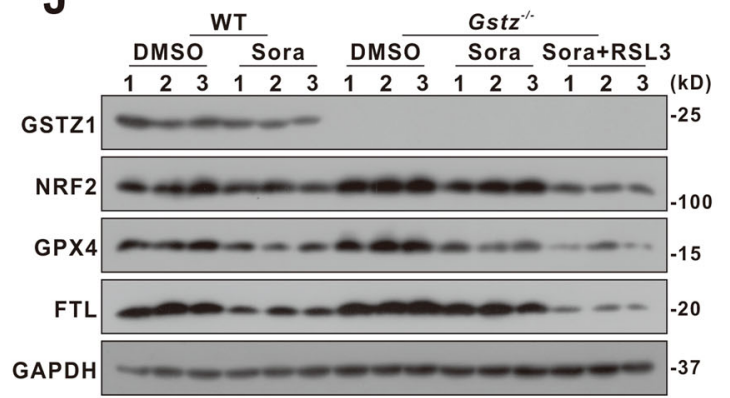

H
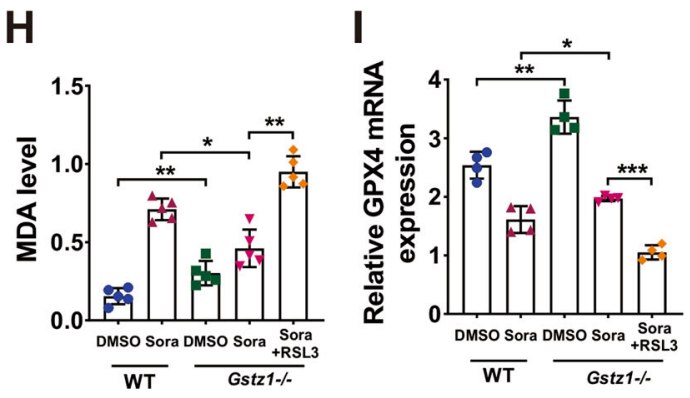

E

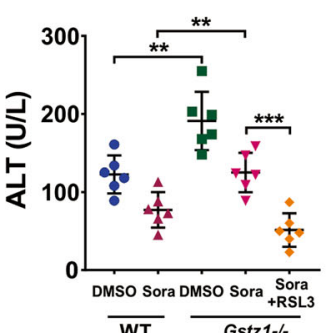

F

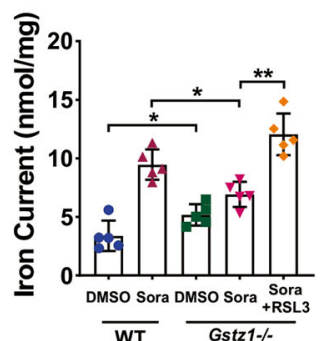

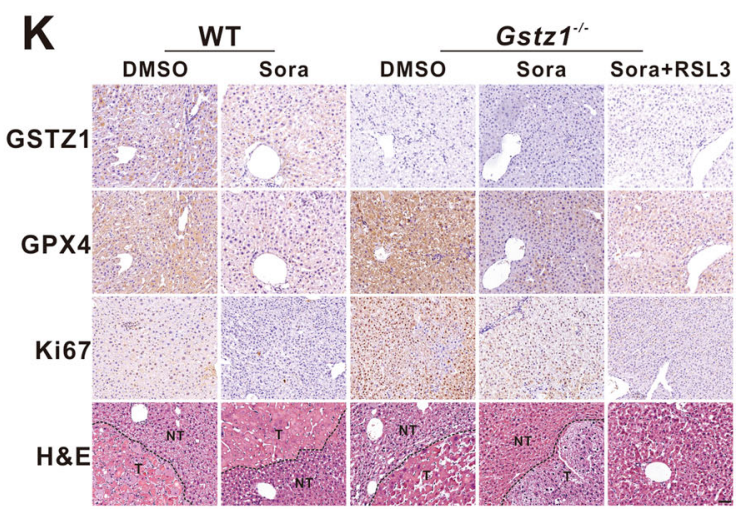

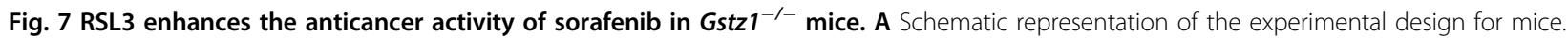
B Gross appearances of liver tumors. The red circles represent tumors. C-E In vivo analyses of liver/body weight ratio (C), tumor numbers (D), and serum alanine aminotransferase (ALT) (E) levels of the five groups. $(n=6)$. F-H Levels of iron $(\mathbf{F})(n=5), 4-\mathrm{HNE}$ modification $(\mathbf{G})(n=3)$ and MDA $(\mathbf{H})$ $(n=5)$ in mice were assayed. I, J mRNA $(\mathbf{I})(n=4)$ and protein $(\mathbf{J})(n=3)$ levels of GPX4, FTL, and SLC7A11 in the liver tumors as assessed using qRTPCR and western blotting, respectively. K Representative H\&E staining and immunohistochemistry images of GSTZ1, GPX4, and Ki67 in hepatic tumors. Scale bar $=50 \mu \mathrm{m}$. For western blotting, $50 \mu \mathrm{g}$ protein was loaded per well. WT wild-type, DEN diethylnitrosamine, $C C{ }_{4}$ carbon tetrachloride, DMSO dimethyl sulfoxide, Sora sorafenib, RSL3 Ras-selective lethal small molecule 3, ALT alanine aminotransferase, 4-HNE 4-hydroxy-2-nonenal, H\&E hematoxylin and eosin. ${ }^{*} p<0.05,{ }^{* *} p<0.01,{ }^{* * *} p<0.001$, Student's $t$-test (two groups) or one-way ANOVA followed by Tukey's tests (five groups). 


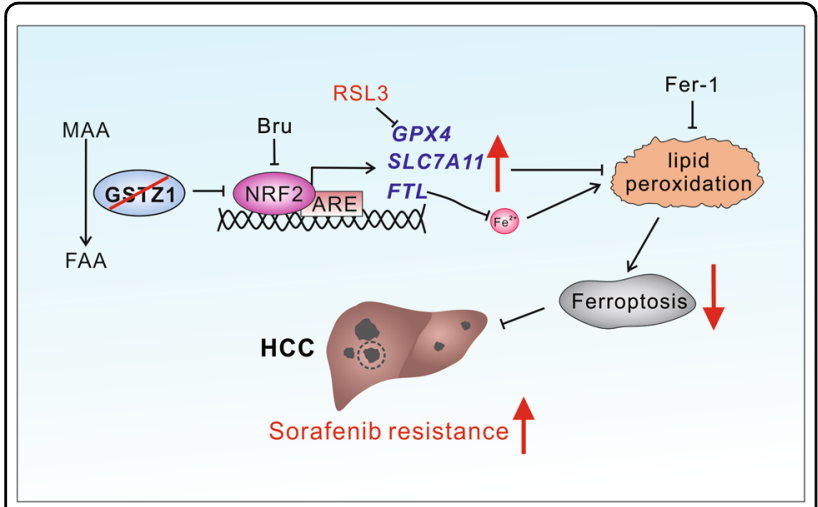

Fig. 8 A proposed model of the resistance of GSTZ1-deficient cells to sorafenib. MAA maleylacetoacetate, FAA fumarylacetoacetate, Bru brusatol, ARE anti-oxidation response element, Fer-1 ferrostatin-1, HCC hepatocellular carcinoma.

has been found to induce ferroptosis by modulating iron metabolism and enhancing the accumulation of lipid peroxidation $^{6,42}$. As homeostatic dysfunction of ferroptosis is believed to be an essential cause of chemoresistance $^{43}$, it is crucial to explore how to enhance the sensitivity of cancer cells to clinical chemotherapy drugs by triggering ferroptosis. In this case, ferroptosis inducer erastin significantly enhances the anticancer activity of cytarabine and doxorubicin in leukemia cells ${ }^{44}$. Erastin also reverses the resistance of ovarian cancer cells to cisplatin ${ }^{45}$. Multiple studies recently verified that sorafenib plays an important role in inducing ferroptosis. Herein, we found that GSTZ1 deficiency aggravated the resistance to sorafenib-induced ferroptosis by preventing iron accumulation and lipid peroxidation production and decreasing the ROS levels. In contrast, GSTZ1 overexpression increased the sensitivity of HCC cells to sorafenib by facilitating ferroptosis in vitro. Consistent with this study, previous studies have reported that sorafenibinduced hepatoma cell death is primarily dependent on triggering ferroptosis by inhibiting SLC7A11/xCT $\mathrm{T}^{10,11,24}$. As such, inducing ferroptosis may be a promising strategy for enhancing the sensitivity of tumor cells to chemotherapy. Haloperidol, a Sigma-1 receptor (S1R) antagonist, promotes sorafenib-induced ferroptotic death by increasing ROS accumulation ${ }^{46}$. Meanwhile, metallothionein-1G silencing (MT-1G) was reported to enhance the sensitivity of hepatoma cells to sorafenib by triggering ferroptosis ${ }^{24}$. Collectively, our findings and those of previous studies suggest that ferroptosis plays an important role in the anti-tumor efficacy of sorafenib. Further, our data indicate that GSTZ1 plays a positive regulatory role in ferroptosis during sorafenib treatment.

Changes in certain metabolic pathways are also involved in the regulation of cell sensitivity to ferroptosis, including coenzyme Q10 consumption ${ }^{47}$, decreased intracellular reducer such as $\mathrm{NAPDH}^{48}$, and altered iron metabolism ${ }^{49}$. Many components of the ferroptosis cascade are target genes of the transcription factor NRF2, indicating the critical role of the NRF2 pathway in mediating ferroptotic response $\mathrm{e}^{30,31,50}$. For example, the inhibition of $\mathrm{p} 62$ KEAP1-NRF2 pathway significantly enhanced the anticancer activity of erastin and sorafenib by inducing ferroptosis in HCC cells in vitro and in vivo ${ }^{26}$. Consistent with previous reports, our results demonstrated that GSTZ1 deficiency markedly reduces sorafenib-induced ferroptotic cell death by increasing the level of NRF2 and ferroptosis-related genes, including GPX4, SLC7A11, and FTL. In contrast, pharmacological- or KEAP1-mediated inhibition of NRF2 increases the sensitivity of GSTZ1deficient cells to sorafenib by enhancing ferroptosis in vitro. Meanwhile, GPX4 is the only reported enzyme that is capable of directly reducing complex phospholipid hydroperoxides and is a downstream target gene of NRF2. Therefore, targeting GPX4 is currently considered a crucial strategy for triggering ferroptosis ${ }^{7,43}$ Mechanistically, we verified that GSTZ1 knockout inhibited sorafenibinduced ferroptosis by activation of the NRF2/GPX4 axis in vitro and in vivo. Moreover, targeting GPX4 using RSL3 in GSTZ1-knockout and SR HCC cells significantly increased iron accumulation, ROS level, and lipid peroxidation production and enhanced sorafenib-induced inhibition of cell proliferation. Importantly, GPX4 inhibition using RSL3 with sorafenib therapy elicited a significant tumor regression in $\mathrm{Gstz1}^{-/-}$mouse models in vivo.

To our best knowledge, this is the first study to explore the role of GSTZ1 in sorafenib resistance in HCC. GSTZ1 deficiency contributes to sorafenib resistance in HCC through activation of the NRF2/GPX4 axis; thus, blocking the NRF2/GPX4 axis may have a therapeutic benefit in HCC patients with GSTZ1 deficiency. Our findings provide new insights into the molecular basis of the role of GSTZ1 in ferroptosis and sorafenib resistance. Moreover, sorafenib combined with RSL3 can synergistically overcome acquired resistance to sorafenib and improve the anticancer efficacy of sorafenib in HCC, thus providing a potentially synergistic combination therapy for HCC.

\section{Materials and methods \\ Cell lines}

Human hepatoma cells SK-Hep1, HepG2, and SNU449 were directly purchased from American Type Culture Collection (ATCC, VA, USA). Huh7 cells were obtained from the Cell Bank of the Chinese Academy of Sciences (Shanghai, China). These cells were cultured in Dulbecco's modified Eagle's medium (SK-Hep1, HepG2, Huh7) or RPMI 1640 medium (SNU449) supplemented with 10\% fetal bovine serum (FBS; Gibco, Rockville, MD, USA), 
100 units $/ \mathrm{mL}$ penicillin, and $100 \mathrm{mg} / \mathrm{mL}$ streptomycin in a humidified incubator at $37^{\circ} \mathrm{C}$ containing $5 \% \mathrm{CO}_{2}$.

\section{Reagents and antibodies}

Erastin (HY-15763), Ferrostatin-1 (HY-100579), Deferoxamine (HY-B0988), and Necrosulfonamide (HY100573) were purchased from MedChemExpress (MCE; Shanghai, China). Sorafenib (S7397), ZVAD-FMK (S7023), Bafilomycin A1 (S1413), and RSL3 (S8155) were obtained from Selleckchem (Houston, TX, USA). Nacetyl-L-cysteine (NAC, S0077) was from Beyotime (Shanghai, China). Brusatol (Bru, MB7292) was obtained from Meilunbio (Dalian, China). Tertiary butylhydroquinone (tBHQ, 112941) was obtained from Sigma (Shanghai, China). Antibodies raised against GPX4 (ab125066), NRF2 (ab62352), 4-HNE (ab46545), NQO1 (ab34173), and $\beta$-actin (ab6276) were obtained from Abcam (Cambridge, MA, USA), anti-SLC7A11 (NB300318) was from Novusbio (Centennial, CO, USA), anti-FTL (10727-1-AP) was from Proteintech (Shanghai, China), and anti-GSTZ1 (GTX106109) was from GeneTex (San Antonio, CA, USA).

\section{Generation of SR cell lines}

To establish SR cells, HepG2 and SNU449 cells were cultured by exposing cells with sorafenib at $5 \%$ of $\mathrm{IC}_{50}$ concentration and the concentration was gradually increased at $10 \%$ of $\mathrm{IC}_{50}$ until the maximum tolerated doses $(10 \mu \mathrm{M})$ was reached. SR cells (HepG2-SR and SNU449-SR) were cultured continuously at $1 \mu \mathrm{M}$ concentration of sorafenib to maintain the acquired resistance.

\section{Quantitative real-time polymerase chain reaction (qRT- PCR)}

Total RNA was isolated from HCC cell lines using TRIzol reagent (Invitrogen, Rockville, MD, USA) according to the manufacturer's instructions. The purified RNA sample was reverse-transcribed into cDNA using the PrimeScript $^{\mathrm{TM}}$ RT Reagent Kit (RR047A, TaKaRa, Tokyo, Japan) with gDNA Eraser. Complementary DNA from cell samples was amplified with the specific primers (Supplementary Table. 1). Briefly, Real-time qPCR was performed to quantity mRNA levels, using the SYBR Green qPCR Master Mix (Bio-Rad, Hercules, CA, USA) following the manufacturer's instructions. The CT values were normalized to that of $\beta$-actin, and the relative mRNA expression levels of genes were calculated using $2^{-\Delta \Delta C t}$ method. Each sample was analyzed in triplicate.

\section{Western blot analysis}

Protein samples from cells and animal tissues were lysed in Cell Lysis Buffer (Beyotime, Jiangsu, China) containing $1 \mathrm{mM}$ of phenylmethanesulfonyl fluoride (PMSF,
Beyotime). The concentration of the protein homogenates was determined using the BCA assay Kit (Dingguo, Beijing, China). Equal volumes of protein samples were separated by SDS-poly acrylamide gel electrophoresis and electro-transferred to PVDF membranes (Millipore, Billerica, MA, USA). After blocked with $5 \%$ non-fat milk dissolved in TBST $(10 \mathrm{mM}$ Tris, $150 \mathrm{mM} \mathrm{NaCl}$, and $0.1 \%$ Tween-20; $\mathrm{pH} 7.6$ ), for $2 \mathrm{~h}$ at room temperature, the membranes were incubated with the primary antibodies overnight at $4{ }^{\circ} \mathrm{C}$. Thereafter, membranes were incubated with the secondary antibodies coupled to horseradish peroxidase (HRP) for $2 \mathrm{~h}$ at room temperature. Protein bands were visualized with enhanced Chemiluminescence substrate Kits (ECL, New Cell \& Molecular Biotech Co, Ltd, China).

\section{Transmission electron microscope assay}

Cells were collected and fixed with $2.5 \%$ glutaraldehyde. Subsequently, cells were post-fixed in $2 \%$ tetroxide and dehydrated through a series of gradient ethanol. Samples were embedded in epoxy resin, cut into thin slices, and placed onto nickel grid. Images were acquired using Hitachi-7500 transmission electron microscope (Hitachi, Tokyo, Japan).

\section{Intracellular ROS measurements}

Cells were planted on coverslips in a 12-well plate, and then treated with the varying concentrations of test compound or drug. After $24 \mathrm{~h}$, cells were incubated at a final concentration of $5 \mu \mathrm{M}$ CellROX® Orange reagent (Life Technologies, Carlsbad, USA) for $30 \mathrm{~min}$ at $37^{\circ} \mathrm{C}$, after which they were washed, dyed with DAPI, mounted with Anti-fade Mounting Medium, and immediately analyzed for fluorescence intensity under Leica Confocal Microscope (TCS SP8, Germany) with a $\times 40$ objective lens.

\section{Measurement of total iron contents in hepatoma cells and liver tissues}

The iron concentration was assessed using the Iron Assay Kit (MAK025; Sigma) according to the manufacturer's protocol. Briefly, tissues $(10 \mathrm{mg})$ or cells $(2 \times$ $10^{6}$ ) were rapidly homogenized in 5 volumes of iron assay buffer. Tissues or cell homogenates were centrifuged at $15,000 \times g$ for $10 \mathrm{~min}$ at $4{ }^{\circ} \mathrm{C}$ and removed insoluble material. To measure total iron, add $50 \mu \mathrm{L}$ samples to $96-$ well plate, increase the volume to $100 \mu \mathrm{L}$ per well with iron assay buffer, and then add $5 \mu \mathrm{L}$ iron reducer to each well to reduce $\mathrm{Fe}^{3+}$ to $\mathrm{Fe}^{2+}$. And then samples were mixed using a horizontal shaker and incubated at $25^{\circ} \mathrm{C}$ for $30 \mathrm{~min}$. Subsequently, $100 \mu \mathrm{L}$ Iron Probe was added and incubated for $1 \mathrm{~h}$ at $25^{\circ} \mathrm{C}$. During each incubation, the plate was kept from light. Thereafter, the absorbance was detected at $593 \mathrm{~nm}$ using a microplate reader. 


\section{Detection of malondialdehyde (MDA)}

Analysis of lipid peroxidation was assessed by quantification of MDA concentration in cell lysates using Lipid Peroxidation MDA Assay Kit (S0131, Beyotime) in accordance with the manufacturer's instructions.

\section{Reduced GSH assay measurement}

Reduced GSH levels were determined using the GSH and GSSG Assay Kit (Beyotime, S0053) following the manufacturer's protocol.

\section{Reporter plasmids and mutagenesis}

Human Nrf2 expression plasmid was constructed by subcloning PCR amplification and inserted it into pcDNA-5Myc vector (a gift from prof. Wei Lv, Shanghai Institute of Nutrition and Health, Chinese Academy of Sciences). Human Gstz1 and Keap1 expression plasmids were constructed by subcloning PCR amplification and inserted into pSEB-3Flag vector (a gift from Dr T-C He, University of Chicago, USA). The Ser to Ala mutant of Gstz1 (S14A) was constructed by sitedirected mutagenesis, with WT Gstz1-3Flag plasmid as a template.

\section{Cell growth curve and cell viability assay}

For cell growth curve analysis, cells were seeded at $1 \times 10^{4}$ cells/well in 96-well microtiter plates with three replicate per group and cultured overnight in a humidified incubator at $37{ }^{\circ} \mathrm{C}$ and $5 \% \mathrm{CO}_{2}$. The plate was scanned and phase-contrast images were acquired after over a series of time points post treatment, and then quantified time-lapse curves were plotted using IncuCyte ZOOM software (Essen BioScience, Ann Arbor, MI, USA).

For cell viability assay, cells were seeded at 1000 cells per well in 96-well plates with fresh medium and analyzed by using the Cell Counting Kit-8 (CCK-8) (CK04, Dojindo, Japan) according to the manufacturer's instructions. The microplates were incubated at $37^{\circ} \mathrm{C}$ for an additional $2 \mathrm{~h}$. Absorbance (Abs) was read at $450 \mathrm{~nm}$ using a microplate reader (Thermo Fisher, USA). The blank group only contained medium, and the cells without any treatment were used as the control group. Herein, cell viability $=($ Abs of the experimental group-Abs of the blank group)/(Abs of the control group-Abs of the blank group) $\times 100 \%$.

\section{Cell growth rate and cell death}

Cell growth was analyzed at $24,48,72$ or $96 \mathrm{~h}$ post culture. The cell growth rate was calculated by dividing the final number of cells by the number of cells seeded at day 0 and expressed as a percentage. Cell death was analyzed by propidium iodide (Invitrogen, USA) staining followed by flow cytometry.

\section{Half-maximal inhibitory concentration assay $\left(\mathrm{IC}_{50}\right)$}

The cells were planted in 96-well plates at $1.0 \times 10^{4}$ cells per well. The corresponding concentrations of sorafenib were given to cells for $24 \mathrm{~h}$. After $24 \mathrm{~h}$, CCK-8 (Dojindo, Japan) was used to measure sorafenib sensitivity at $450 \mathrm{~nm}$ using a microplate reader (Thermo Fisher, USA) after incubating for $2 \mathrm{~h}$ at $37^{\circ} \mathrm{C}$.

\section{Animal experiments}

Heterozygous 129-Gstz1 ${ }^{\mathrm{tm} 1 \mathrm{mfc}} / \mathrm{Cnbc}$ mice (EM: 04481) were obtained from the European Mouse Mutant Archive and were crossed to breed wild-type (WT) and Gstz1 $1^{-/-}$ mice. All mice were maintained under individual ventilation cages conditions in the laboratory animal center of Chongqing Medical University. For subsequent studies, mice were divided into five groups as follows: WT + DMSO (control), WT + Sora, Gstz1 ${ }^{-1-}+\mathrm{DMSO}$, $\mathrm{Gstz1}^{-1-}+$ Sora, and $G s t z 1^{-1-}+$ Sora + RSL3. Each group included three male and three female mice. At 2 weeks of age, all mice were administered an intraperitoneal injection of diethylnitrosamine (DEN; Sigma, St. Louis, MO, USA) at a dose of $75 \mathrm{mg} / \mathrm{kg}$. At the third week, the mice were intraperitoneally administered carbon tetrachloride $\left(\mathrm{CCl}_{4} ;\right.$ Macklin, Shanghai, China) at $2 \mathrm{ml} / \mathrm{kg}$ twice a week for 12 weeks. In the WT + Sora and Gstz1 ${ }^{-1}$ ${ }^{-}+$Sora group, the mice at 22 weeks were administered intraperitoneally sorafenib $(30 \mathrm{mg} / \mathrm{kg})$ every 2 days for 4 weeks until euthanasia. In the $\mathrm{Gstz1}^{-1-}+$ Sora+RSL3 group, in addition to sorafenib administration as described above, the mice were injected intraperitoneally with RSL3 $(10 \mathrm{mg} / \mathrm{kg})$ every 2 days for 4 weeks at the same weeks. Body weight of each mice was measured every week and retro-orbital blood was collected before sacrifice. All mice were euthanized at 26 weeks of age. The liver weight and number of liver tumors were measured. Protein and mRNAs levels of hepatic tumors were detected by western blotting and qRT-PCR analysis, respectively. The intrahepatic Iron and MDA levels were measured with Iron Assay Kit and MDA Assay Kit, respectively. Samples of liver tumor were collected for further study or fixed with $4 \%$ paraformaldehyde, embedded in paraffin, and sectioned for hematoxylineosin staining (H\&E) and immunohistochemistry. All animal procedures were approved by the Research Ethics Committee of Chongqing Medical University (reference number: 2017010).

\section{Statistical analysis}

All experiments were repeated independently with similar results at least three times. Statistical analysis and data plotting were performed using GraphPad Prism 7 (GraphPad Software, USA). All data were presented as mean \pm standard deviation (SD) values. Unless mentioned otherwise, comparisons between two groups were 
performed by Student's $t$-test, and Multiple-group comparisons were performed by the one-way ANOVA analysis with Scheffe post-hoc test. $p<0.05$ was considered statistically significant.

\section{Acknowledgements}

We wish to thank Dr. T.-C. He (University of Chicago, USA) for providing the plasmids pAdEasy system, and Prof. Ding Xue (Tsinghua University) for supplying the CRISPR/Cas9 system.

\section{Author contributions}

A.L.H., N.T., and K.W. conceived the study and designed the experiments. Q.J.W. and B.C. performed most experiments and analyzed the data. Q.X. performed TEM assay. Q.Z.G. assisted with animal experiments. Q.J.W., N.T., and K.W. drafted and edited the manuscript with all authors providing feedback.

\section{Funding}

This work was supported by the China National Natural Science Foundation (grant no. 81872270, 82072286, 82073251), the Natural Science Foundation Project of Chongqing (cstc2018jcyjAX0254, cstc2019jcyj-msxmX0587), the Major National S\&T program (2017ZX10202203-004), the Scientific Research Innovation Project for Postgraduate in Chongqing (grant no. CYS19192), the Science and Technology Research Program of Chongqing Municipal Education Commission (KJZD-M202000401, KJQN201900429), and the Kuanren Talents Program of the second affiliated hospital of Chongqing Medical University.

\section{Data availability}

The datasets used and analyzed during the current study are available from the corresponding author on reasonable request.

\section{Ethics statement}

This study was approved by the Research Ethics Committee of Chongqing Medical University (reference number: 2017010).

\section{Conflict of interest}

The authors declare no competing interests.

\section{Publisher's note}

Springer Nature remains neutral with regard to jurisdictional claims in published maps and institutional affiliations.

Supplementary information The online version contains supplementary material available at https://doi.org/10.1038/s41419-021-03718-4.

Received: 3 January 2021 Revised: 15 April 2021 Accepted: 15 April 2021 Published online: 30 April 2021

\section{References}

1. Bray, F. et al. Global cancer statistics 2018: GLOBOCAN estimates of incidence and mortality worldwide for 36 cancers in 185 countries. CA 68, 394-424 (2018).

2. Yang, J. D. et al. A global view of hepatocellular carcinoma: trends, risk, prevention and management. Nat. Rev. Gastroenterol. Hepatol. 16, 589-604 (2019).

3. Cheng, A.-L. et al. Efficacy and safety of sorafenib in patients in the Asia-Pacific region with advanced hepatocellular carcinoma: a phase III randomised, double-blind, placebo-controlled trial. Lancet Oncol. 10, 25-34 (2009).

4. Raoul, J.-L. et al. Systemic therapy for intermediate and advanced hepatocellular carcinoma: Sorafenib and beyond. Cancer Treat. Rev. 68, 16-24 (2018).

5. Dixon, S. J. et al. Ferroptosis: an iron-dependent form of nonapoptotic cell death. Cell 149, 1060-1072 (2012).

6. Stockwell, B. R. et al. Ferroptosis: a regulated cell death nexus linking metabolism, redox biology, and disease. Cell 171, 273-285 (2017).

7. Forcina, G. C. \& Dixon, S. J. GPX4 at the crossroads of lipid homeostasis and ferroptosis. Proteomics 19, 1800311 (2019).
8. Lei, P., Bai, T. \& Sun, Y. Mechanisms of ferroptosis and relations with regulated cell death: a review. Front. Physiol. 10, 139 (2019).

9. Nie, J., Lin, B., Zhou, M., Wu, L. \& Zheng, T. Role of ferroptosis in hepatocellular carcinoma. J. Cancer Res. Clin. Oncol. 144, 2329-2337 (2018).

10. Lachaier, E. et al. Sorafenib induces ferroptosis in human cancer cell lines originating from different solid tumors. Anticancer Res. 34, 6417-6422 (2014).

11. Louandre, C. et al. Iron-dependent cell death of hepatocellular carcinoma cells exposed to sorafenib: iron-dependent cytotoxicity of sorafenib. Int. J. Cancer 133, 1732-1742 (2013).

12. Townsend, D. M. \& Tew, K. D. The role of glutathione-S-transferase in anticancer drug resistance. Oncogene 22, 7369-7375 (2003).

13. Zhang, J. et al. Altered redox regulation and S-glutathionylation of BiP contribute to bortezomib resistance in multiple myeloma. Free Radic. Biol. Med. $160,755-767(2020)$

14. Henderson, C. J., McLaren, A. W. \& Wolf, C. R. In vivo regulation of human glutathione transferase GSTP by chemopreventive agents. Cancer Res. $\mathbf{7 4}$, 4378-4387 (2014).

15. Wang, X. \& Huang, Z. H. Predictive potential role of glutathione S-transferase polymorphisms in the prognosis of breast cancer. Genet Mol. Res. 14, 10236-10241 (2015)

16. Tew, K. D. Glutathione-associated enzymes in anticancer drug resistance Cancer Res. 76, 7-9 (2016).

17. Rocha, G., da, G., Oliveira, R. R., Kaplan, M. A. C. \& Gattass, C. R. 33-Acetyl tormentic acid reverts MRP1/ABCC1 mediated cancer resistance through modulation of intracellular levels of GSH and inhibition of GST activity. Eur. J. Pharmacol. 741, 140-149 (2014).

18. Fernández-Cañón, J. M. \& Peñalva, M. A. Characterization of a fungal maleylacetoacetate isomerase gene and identification of its human homologue. J. Biol. Chem. 273, 329-337 (1998).

19. Yang, F. et al. GSTZ1-1 deficiency activates NRF2/IGF1R axis in HCC via accumulation of oncometabolite succinylacetone. EMBO J. https://doi.org/ 10.15252/embj.2019101964 (2019).

20. Li, J. et al. GSTZ1 deficiency promotes hepatocellular carcinoma proliferation via activation of the KEAP1/NRF2 pathway. J. Exp. Clin. Cancer Res. 38, 438 (2019).

21. van Malenstein, H. et al. Long-term exposure to sorafenib of liver cancer cells induces resistance with epithelial-to-mesenchymal transition, increased invasion and risk of rebound growth. Cancer Lett. 329, 74-83 (2013).

22. Ye, Z et al. Abrogation of ARF6 promotes RSL3-induced ferroptosis and mitigates gemcitabine resistance in pancreatic cancer cells. Am. J. Cancer Res. 10, 1182-1193 (2020)

23. Li, Y. et al. Erastin/sorafenib induces cisplatin-resistant non-small cell lung cancer cell ferroptosis through inhibition of the Nrf2/xCT pathway. Oncol. Lett. 19, 323-333 (2020)

24. Sun, $X$. et al. Metallothionein-1G facilitates sorafenib resistance through inhibition of ferroptosis. Hepatology 64, 488-500 (2016).

25. Dixon, S. J. et al. Pharmacological inhibition of cystine-glutamate exchange induces endoplasmic reticulum stress and ferroptosis. elife 3, e02523 (2014).

26. Sun, X. et al. Activation of the p62-Keap1-NRF2 pathway protects against ferroptosis in hepatocellular carcinoma cells: hepatobiliary malignancies. Hepatology 63, 173-184 (2016)

27. Li, D. \& Li, Y. The interaction between ferroptosis and lipid metabolism in cancer. Sig. Transduct. Target Ther. 5, 108 (2020).

28. Board, P. G. et al. Clarification of the role of key active site residues of glutathione transferase Zeta/maleylacetoacetate isomerase by a new spectrophotometric technique. Biochem. J. 374, 731-737 (2003).

29. Kobayashi, A. et al. Oxidative stress sensor keap1 functions as an adaptor for Cul3-based E3 ligase to regulate proteasomal degradation of Nrf2. MCB 24, 7130-7139 (2004)

30. Dai, C. et al. Transcription factors in ferroptotic cell death. Cancer Gene Ther. 27 645-656 (2020).

31. Dodson, M., Castro-Portuguez, R. \& Zhang, D. D. NRF2 plays a critical role in mitigating lipid peroxidation and ferroptosis. Redox Biol. 23, 101107 (2019).

32. Venook, A. P., Papandreou, C., Furuse, J. \& Ladrón de Guevara, L. The incidence and epidemiology of hepatocellular carcinoma: a global and regional perspective. Oncologist 15, 5-13 (2010).

33. Llovet, J. M., Montal, R., Sia, D. \& Finn, R. S. Molecular therapies and precision medicine for hepatocellular carcinoma. Nat. Rev. Clin. Oncol. 15, 599-616 (2018).

34. Pereira, D., Assis, J., Gomes, M., Nogueira, A. \& Medeiros, R. Improvement of a predictive model in ovarian cancer patients submitted to platinum-based 
chemotherapy: implications of a GST activity profile. Eur. J. Clin. Pharm. $\mathbf{7 2}$ 545-553 (2016).

35. Sawers, L. et al. Glutathione S-transferase P1 (GSTP1) directly influences platinum drug chemosensitivity in ovarian tumour cell lines. Br. J. Cancer $\mathbf{1 1 1}$ 1150-1158 (2014).

36. Singh, R. R., Mohammad, J., Orr, M. \& Reindl, K. M. Glutathione S-transferase pi-1 knockdown reduces pancreatic ductal adenocarcinoma growth by activating oxidative stress response pathways. Cancers 12, 1501 (2020).

37. Tong, X., Zhao, J., Zhang, Y., Mu, P. \& Wang, X. Expression levels of MRP1, GST$\pi$, and GSK3 $\beta$ in ovarian cancer and the relationship with drug resistance and prognosis of patients. Oncol. Lett. 18, 22-28 (2019).

38. Wang, Q. Expression of P-gp, MRP, LRP, GST- $\pi$ and Topolla and intrinsic resistance in human lung cancer cell lines. Oncol. Rep. 26, 1081-1089 (2011).

39. Zou, M. et al. Glutathione S-transferase isozyme alpha 1 is predominantly involved in the cisplatin resistance of common types of solid cancer. Oncol. Rep. 41, 989-998 (2019).

40. Manz, D. H., Blanchette, N. L., Paul, B. T., Torti, F. M. \& Torti, S. V. Iron and cancer: recent insights: iron and cancer. Ann. NY Acad. Sci. 1368, 149-161 (2016).

41. Torti, S. V. \& Torti, F. M. Iron and cancer: more ore to be mined. Nat. Rev. Cancer 13, 342-355 (2013)

42. Hassannia, B., Vandenabeele, P. \& Vanden Berghe, T. Targeting ferroptosis to iron out cancer. Cancer Cell 35, 830-849 (2019).
43. Friedmann Angeli, J. P., Krysko, D. V. \& Conrad, M. Ferroptosis at the crossroads of cancer-acquired drug resistance and immune evasion. Nat. Rev. Cancer 19, 405-414 (2019).

44. $\mathrm{Yu}, \mathrm{Y}$. et al. The ferroptosis inducer erastin enhances sensitivity of acute myeloid leukemia cells to chemotherapeutic agents. Mol. Cell. Oncol. 2, e1054549 (2015).

45. Sato, M. et al. The ferroptosis inducer erastin irreversibly inhibits system xcand synergizes with cisplatin to increase cisplatin's cytotoxicity in cancer cells. Sci. Rep. 8, 968 (2018).

46. Bai, T. et al. Haloperidol, a sigma receptor 1 antagonist, promotes ferroptosis in hepatocellular carcinoma cells.nbib. Biochem. Biophys. Res. Commun. 491 919-925 (2017).

47. Shimada, K. et al. Global survey of cell death mechanisms reveals metabolic regulation of ferroptosis. Nat. Chem. Biol. 12, 497-503 (2016).

48. Shimada, K. Hayano, M., Pagano, N. C. \& Stockwell, B. R. Cell-line selectivity improves the predictive power of pharmacogenomic analyses and helps identify NADPH as biomarker for ferroptosis sensitivity. Cell Chem. Biol. 23, 225-235 (2016)

49. Bogdan, A. R., Miyazawa, M., Hashimoto, K. \& Tsuji, Y. Regulators of iron homeostasis: new players in metabolism, cell death, and disease. Trends Biochem. Sci. 41, 274-286 (2016).

50. Anandhan, A., Dodson, M., Schmidlin, C. J., Liu, P. \& Zhang, D. D. Breakdown of an ironclad defense system: the critical role of NRF2 in mediating ferroptosis. Cell Chem. Biol. 27, 436-447 (2020). 